\title{
A simulation study on the phase behavior of hard rhombic platelets
}

N. Tasios and M. Dijkstra

Citation: The Journal of Chemical Physics 146, 144901 (2017); doi: 10.1063/1.4979517

View online: http://dx.doi.org/10.1063/1.4979517

View Table of Contents: http://aip.scitation.org/toc/jcp/146/14

Published by the American Institute of Physics

\section{Articles you may be interested in}

Transferable ionic parameters for first-principles Poisson-Boltzmann solvation calculations: Neutral solutes in aqueous monovalent salt solutions

The Journal of Chemical Physics 146, 134103134103 (2017); 10.1063/1.4978850

Experimental prototype of a spin-wave majority gate

The Journal of Chemical Physics 110, 152401152401 (2017); 10.1063/1.4979840

Optical absorbances of Gd3Ga5O12 single crystals under shock compression to $211 \mathrm{GPa}$

The Journal of Chemical Physics 121, 145901145901 (2017); 10.1063/1.4979634

Perspective: Dissipative particle dynamics

The Journal of Chemical Physics 146, 150901150901 (2017); 10.1063/1.4979514

Wireless actuation of piezoelectric coupled micromembrane using radio frequency magnetic field for biomedical applications

The Journal of Chemical Physics 121, 134501134501 (2017); 10.1063/1.4979255

Bright narrowband biphoton generation from a hot rubidium atomic vapor cell

The Journal of Chemical Physics 110, 161101161101 (2017); 10.1063/1.4980073

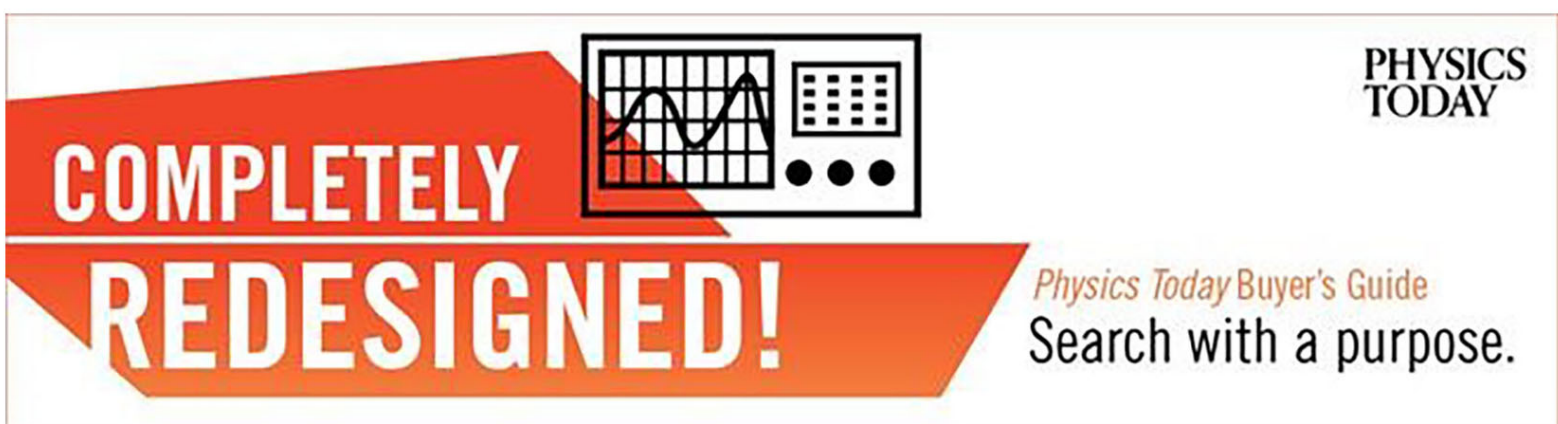




\title{
A simulation study on the phase behavior of hard rhombic platelets
}

\author{
N. Tasios and M. Dijkstraa) \\ Soft Condensed Matter, Debye Institute for Nanomaterials Science, Utrecht University, \\ Princetonplein 1, 3584 CC Utrecht, The Netherlands
}

(Received 21 December 2016; accepted 2 March 2017; published online 10 April 2017)

\begin{abstract}
Using Monte Carlo simulations, we investigate the phase behavior of hard rhombic platelets as a function of the thickness of the platelets, $T$. The phase diagram displays a columnar phase and a crystal phase in which the platelets are stacked in columns that are arranged in a two-dimensional lattice. We find that the shape of the platelets determines the symmetry of the two-dimensional lattice, i.e., rhombic platelets form an oblique columnar phase and a simple monoclinic crystal phase. For sufficiently thick platelets, i.e., for a thickness-to-length ratio $T / L>0.17$, we find only an isotropic fluid, an oblique columnar phase, and a monoclinic crystal phase. Surprisingly, for an intermediate plate thickness, $0.083<T / L<0.17$, we also find a region in between the isotropic (or nematic) phase and the columnar phase, where the smectic phase is stable. For sufficiently thin platelets, $T / L<0.13$, the phase diagram displays a nematic phase. With the exception of the smectic phase, our results resemble the phase behavior of discotic particles. Our results may guide the synthesis and future experiments on rhombic nanoplatelets. Published by AIP Publishing. [http://dx.doi.org/10.1063/1.4979517]
\end{abstract}

\section{INTRODUCTION}

With the advancement of technology and the prevalence of various display devices, liquid crystal displays (LCDs) have played an increasingly important role in our everyday lives. LCDs use the light modulating properties of liquid crystals to display complex images, as such; the study of liquid crystals has never been more important and relevant than at the present age. Whereas normal periodic crystals exhibit long-range positional and orientational order in all spatial dimensions, liquid crystals exhibit long-range orientational and positional order in only two or less spatial dimensions.

Hard-particle models, i.e., models in which the particles interact only by their excluded volume, are particularly important in the study of liquid crystals because they can be used to test molecular theories for the properties of liquid crystals, and they provide a starting point for thermodynamic perturbation theories. The study of such models goes all the way back to 1949 , when Onsager ${ }^{1}$ predicted theoretically that a system of infinitely thin rods or a system of infinitely thin platelets exhibits a first-order phase transition from an isotropic phase to a nematic phase, which is driven purely by excluded-volume interactions. The first time a hard particle model was used to simulate a liquid crystal was in 1972 by Vieillard-Baron, ${ }^{2}$ who investigated the phase behavior of a two-dimensional system of hard ellipses. Due to the computational restrictions of those times, it was not until 1985 that the phase behavior of the full three-dimensional system of ellipsoids was mapped out by Frenkel and Mulder, ${ }^{3}$ revealing isotropic, nematic, crystal, and plastic crystal phases. In 1997, the phase diagram of hard spherocylinders was determined by means of free-energy calculations using Monte Carlo simulations and was found to

\footnotetext{
a) Electronic mail: m.dijkstra@uu.nl
}

exhibit an isotropic, nematic, (plastic) crystal but also a smectic phase. $^{4}$

Frenkel and Eppenga ${ }^{5,6}$ studied the limit of infinitely thin cylinders, or disks, in 1982, finding a first-order isotropicnematic phase transition, and compared it with Onsager theory. ${ }^{1}$ Later, in 1992, Veerman and Frenkel ${ }^{7}$ studied a model for hard platelets, using a sphere truncated by two symmetrically opposite parallel planes, and observed a first-order phase transition from a nematic to a columnar phase which was later also confirmed for infinitely thin disks. ${ }^{8}$ In addition, they found a so-called cubatic phase which turns out to be metastable with respect to the columnar phase in later studies. ${ }^{9-11}$ The phase diagram of hard oblate spherocylinders, i.e., a model system of discotic-like particles, was determined as a function of the plate thickness by Marechal et al. and exhibited isotropic, nematic, columnar, and two crystal phases. Marechal et al. also studied models of hard platelets with different degrees of roundness ${ }^{10}$ focusing on the "devitrification" process from a metastable cubatic to a columnar phase. More recently, a large class of polyhedral-shaped particles has been investigated, that exhibit plastic crystal and liquid crystal phases but also intriguing crystal phases. ${ }^{12-17}$ Although plastic-crystal forming hard particles have been studied since $1985,3,18-20$ the more "exotic" states, such as quasi-crystals, ${ }^{12,21}$ biaxial nematics, ${ }^{22-25}$ and even chiral phases, ${ }^{26}$ have only been studied very recently, which indicates ${ }^{27}$ that the study of hard-particle systems is far from over.

There is an additional reason why hard-particle models have become important as of late, and that is because a wide variety of these systems are now experimentally available. Hard-sphere colloidal particles have been synthesized for over five decades now, ${ }^{28,29}$ and various polyhedral-shaped particles have also been synthesized recently. ${ }^{130-48}$

In this work, we focus on the phase behavior of particles of a specific polyhedral shape known as rhombohedron. The 


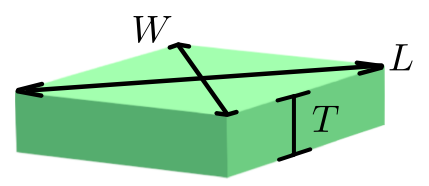

FIG. 1. A rhombic platelet. The three lines denote the characteristic dimensions of the platelet, with $T$ being the thickness, $W$ being the width or short axis length, and $L$ the long axis length. In our simulations, we fix $W / L=2 / 3$.

work is motivated by the recent synthesis of nano-platelets of this shape by Murray et al. ${ }^{45,48}$ and is focused on shapes with a small aspect ratio. For convenience, we refer to these particles as "rhombic platelets." Current research on the selfassembly of these particles ${ }^{48}$ has focused on two-dimensional planar geometries. Instead, we explore the self-assembly of the three-dimensional bulk system, which could prove useful for future experimental studies. We generally expect the phase behavior of platelets of different shapes to be qualitatively similar, i.e., we should at least find nematic, columnar, and crystal phases at the appropriate aspect ratios.

This paper is organized as follows. In Section II we discuss the hard-particle model, and in Section III the computational methods used to simulate and characterize the different phases of the rhombic platelets. In Section IV we present our results, which are focused on the phase behavior of these flat particles. Finally, in Section V we summarize the results and discuss possible future ventures.

\section{MODEL}

We study the phase behavior and structure of a system consisting of hard rhombic platelets using Monte Carlo simulations. To this end, we model each particle as a rhombohedron which is basically a rhombus extruded in the third dimension. In Figure 1 we show a typical rhombic platelet along with three lines, denoting the characteristic dimensions of the platelet, with $T$ being the thickness, $W$ being the short axis length, and $L$ the long axis length. Two-dimensional rhombi can tile space, admitting either a tiling where all platelets are parallel or a socalled rhombille tiling, ${ }^{49}$ with the former being the favorable one as shown in simulations. ${ }^{48}$ Rhombic platelets can also tile the three-dimensional space by stacking different $2 \mathrm{D}$ tilings on top of each other.

Here, we simulate hard rhombic platelets that interact solely through excluded volume interactions, which means that platelets cannot overlap at any point in the simulation. In our simulations, we fix $W / L=2 / 3$, which is close to the experimental value of recently synthesized $\mathrm{GdF}_{3}$ nanoplatelets, ${ }^{45}$ and investigate the phase behavior as a function of the reduced thickness, $T / L$.

\section{METHODS}

We perform Monte Carlo (MC) simulations in the NPT ensemble, i.e., simulations where the number of particles, $N$, pressure $P$, and temperature $T$ are fixed. We employ periodic boundary conditions along all three dimensions. We change the volume independently in each dimension so that the simulation box can accommodate the different phases, thereby avoiding severe finite size effects. We use the cluster algorithm introduced by Almarza ${ }^{50}$ for performing the volume change move. As in the original algorithm, in order to construct clusters, we first introduce bonds between pairs of particles. The probability of generating a bond between two particles depends on the shortest surface-to-surface distance, $s$, and reads

$$
b(s)=\left\{\begin{array}{cl}
1-\left(\frac{s}{\delta}\right)^{2}, & s<\delta, \\
0, & s \geq \delta,
\end{array}\right.
$$

where $\delta$ is the cutoff distance which is sufficiently small that percolation is avoided. For calculating the shortest surfaceto-surface distance, $s$, as well as overlaps between pairs of particles, we use the Gilbert Johnson Keerthi (GJK) algorithm, ${ }^{51}$ which can be employed for arbitrary convex particle shapes. Each Monte Carlo cycle consists of $N$ attempts to translate a random particle, $N$ attempts to rotate a random particle, and one attempt to change the volume of the simulation box. Configurations were initialized in an oblique crystal phase (an example of this phase is shown in Figure 4) and equilibrated for $\sim 5 \cdot 10^{6} \mathrm{MC}$ cycles. The equilibrium packing fraction, $\eta$, was calculated as an average over the last $\sim 10^{5} \mathrm{MC}$ cycles.

To determine the isotropic-nematic $(I-N)$, nematicsmectic $(\mathrm{N}-\mathrm{Sm})$, and smectic-columnar/crystal $(\mathrm{Sm}-\mathrm{C} / \mathrm{X})$ coexistence packing fractions, $\eta$, we study the discontinuities in the equations of state. The equations of state are determined by expanding from the space-filling rhombic crystal phase. Furthermore, we calculate the nematic and smectic order parameters. The nematic order parameter, $S$, and nematic director, $\boldsymbol{n}$, are identified as the largest eigenvalue, and corresponding eigenvector, of the nematic order parameter tensor, ${ }^{52}$

$$
Q_{\alpha \beta}=\frac{1}{N} \sum_{i=1}^{N}\left[\frac{3}{2} u_{i \alpha} \cdot u_{i \beta}-\frac{1}{2} \delta_{\alpha \beta}\right],
$$

where $u_{i \alpha}$ is the $\alpha$-th component of the unit vector describing the orientation of a particular axis of platelet $i, N$ is the number of particles, and $\delta_{\alpha \beta}$ is the Kronecker delta. The smectic order parameter, $\tau$, is calculated along the nematic director, $\boldsymbol{n}$, and is given by ${ }^{53-56}$

$$
\tau=\max _{l}\left|\sum_{j=1}^{N} e^{2 \pi i r_{j} \cdot n / l}\right|,
$$

where $\boldsymbol{r}_{j}$ is the position of the $j$ th platelet, and the value of $l \in R$ that maximizes the above expression is identified as the layer spacing. Additionally, we calculate the diffraction patterns along the nematic director corresponding to the long particle axis, i.e., the $L$-axis. This is done by projecting the particle positions on the plane defined by the nematic directors of the $T$ and $W$ particle axes and subsequently calculating the Fourier transform of a two-dimensional histogram of the projected particle positions.

\section{RESULTS}

In our Monte Carlo study, we find that hard rhombic platelets with a width-to-length ratio $W / L=2 / 3$ exhibit a rich phase behavior; the platelets can transition through up to five different phases, depending on the reduced thickness, $T / L$. In Figure 2(a), we have plotted the equation of state $\left(\beta P V_{p}\right.$ vs $\eta$, 

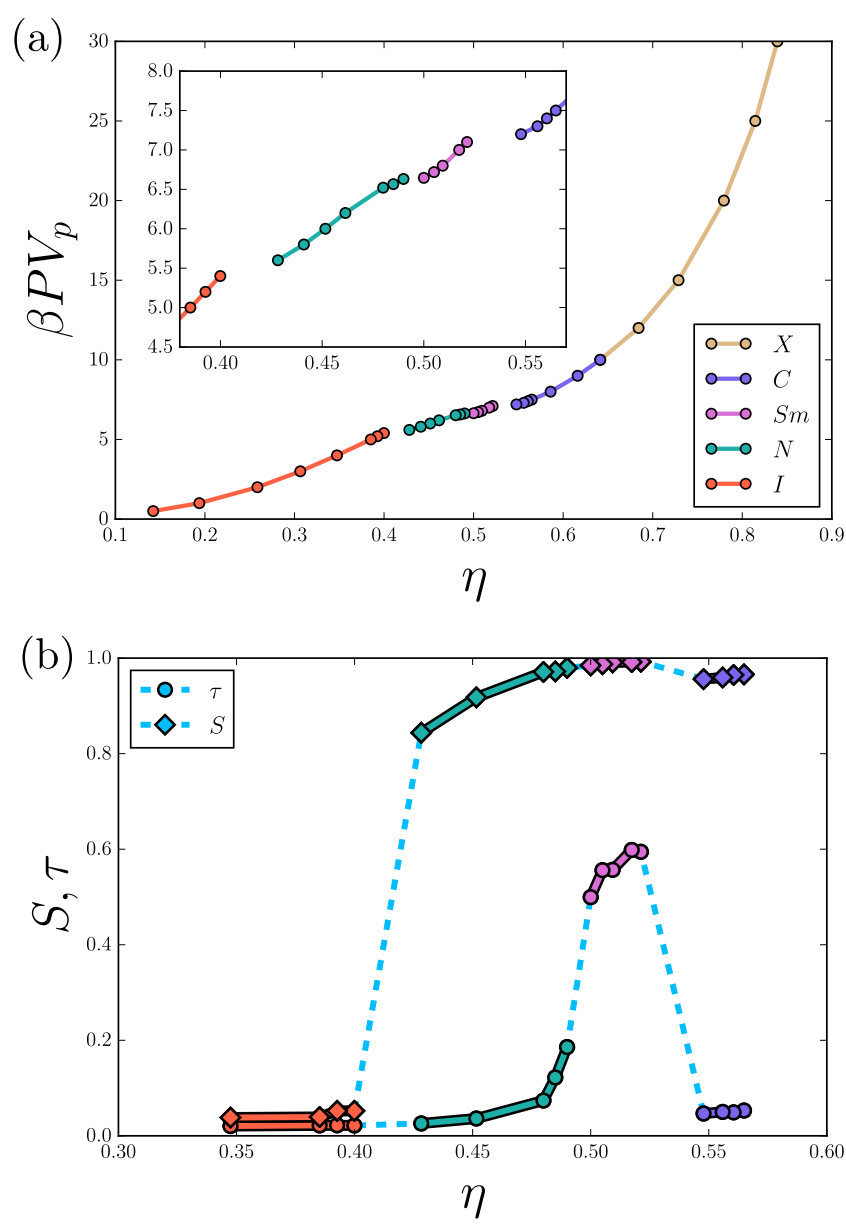

FIG. 2. (a) Equation of state $\left(\beta P V_{p}\right.$ vs $\left.\eta\right)$ for rhombic platelets with reduced width $W / L=2 / 3$ and thickness $T / L=1 / 9$. The different points are colored according to the respective phase. Orange: isotropic phase $(I)$, green: nematic phase $(N)$, pink: smectic phase $(S m)$, purple: columnar phase $(C)$, brown: rhombic crystal phase $(X)$. In the inset, a zoomed-in region of $\eta \in(0.38,0.57)$ is plotted showing more clearly the jumps associated with the three first-order phase transitions (namely, $\mathrm{I}-\mathrm{N}, \mathrm{N}-\mathrm{Sm}$, and $\mathrm{Sm}-\mathrm{C}$ ). (b) Nematic, $\mathrm{S}$, and smectic, $\tau$, order parameters for the same rhombic platelets as in (a). The nematic order parameter is plotted as circles, while the smectic order parameter is plotted as diamonds. The different points are colored according to the color-coding in (a).

where $\beta=1 /\left(k_{B} T\right)$ with $k_{B}$ the Boltzmann constant and $V_{p}$ is the particle volume) for platelets with reduced thickness $T / L=1 / 9$. The colors denote the thermodynamic phase of the respective state point. More specifically, the isotropic phase $(I)$ is represented by orange, the nematic phase $(N)$ by green, the smectic phase $(\mathrm{Sm})$ by pink, the columnar $(\mathrm{C})$ by purple, and the rhombic crystal phase is represented as brown. To appreciate the fine detail of the equation of state, we plot a magnified version for packing fractions $\eta \in(0.38,0.57)$, in the inset, showing the jumps associated with the three firstorder phase transitions ( $I-N, N-S m$, and $S m-C)$. This is further underpinned in Figure 2(b), where we plot the nematic, $S$, and smectic, $\tau$, order parameters, using once more the same colorcoding. It can be clearly seen that the nematic order parameter, $S$, shows a jump at a packing fraction $\eta \approx 0.4$, clearly indicating the orientational alignment of the platelets along the nematic director. On the other hand, the smectic order parameter, $\tau$, clearly shows a jump at a packing fraction $\eta \approx 0.5$. The smectic order parameter jumps back to $\sim 0$ in the columnar phase at a packing fraction $\eta \approx 0.55$.
We now turn our attention to the structure of these phases. To this end, we present configurations, along with the corresponding diffraction patterns, in Figures 3 and 4, for platelets with reduced thickness $T / L=1 / 9$. In the configurations, the particles are colored according to the absolute direction of the short axis-it is easily seen that with the exception of the isotropic phase, as shown in Figure 3(a), all phases are orientationally ordered along the short particle axis, i.e., along the $T$-axis. From the configuration in Figure 3(b) and the corresponding diffraction pattern, we observe that the nematic phase shows only weak positional order of the platelets along the nematic director, which is enhanced and clearly pronounced in the smectic phase. In the columnar phase, as shown in Figure 4(b), we find that the particles form stacks which are arranged in a two-dimensional oblique lattice. We thus find a phase transition from the smectic phase, with one-dimensional positional order and strong orientational order along the nematic director, to a columnar phase with two-dimensional positional order and with additional weak orientational order in the direction perpendicular to the nematic director, as can be seen from the diffraction patterns and configurations.

Combining the information from the equations of state, the order parameters, $S$ and $\tau$, and the diffraction patterns, we determine the phase diagram as a function of plate thickness, $T / L$, which is shown in Figure 5. The hatched blue region denotes the region where the equation of state shows a jump, while the solid-colored regions represent the different phases according to the color-coding used in Figure 2. From the phase diagram, we see that for sufficiently thick platelets, $T / L>0.17$, only the isotropic, columnar, and rhombic crystal phases are present. For thinner platelets, we find a narrow regime where the smectic phase is stable, which is easy to miss. Upon decreasing $T / L$ further, the isotropic region becomes smaller, and the nematic phase takes over. For thickness $0.083<T / L<0.133$, the rhombic platelets experience,

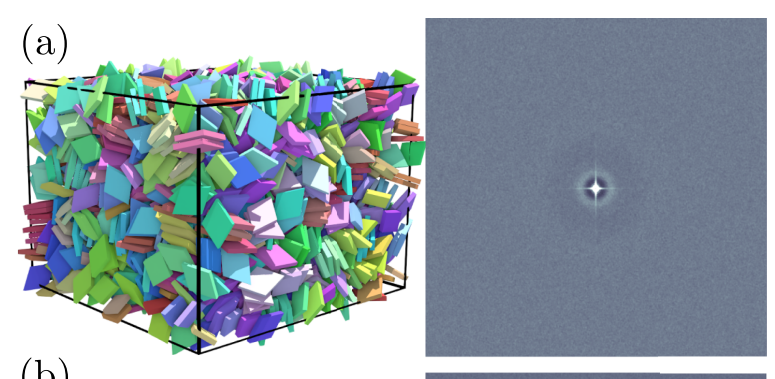

(b)

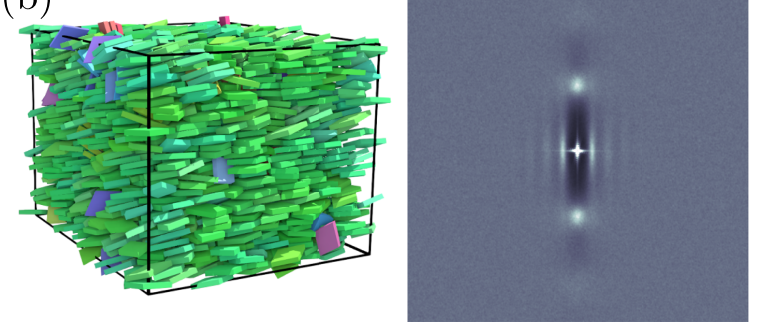

FIG. 3. Left: Representative configurations of hard rhombic platelets with reduced width $W / L=2 / 3$ and thickness $T / L=1 / 9$ for the isotropic (a) and nematic (b) phases. The particles are colored according to the absolute value of the direction of their short axis. Right: Diffraction patterns corresponding to the phases to the left, with the vertical axis being along the $T$-nematic director and the horizontal axis perpendicular to it. 
(a)

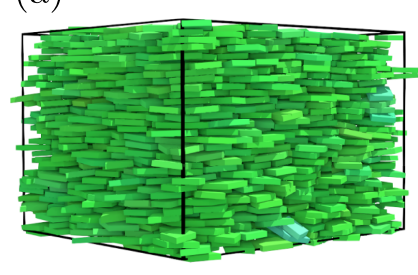

(b)

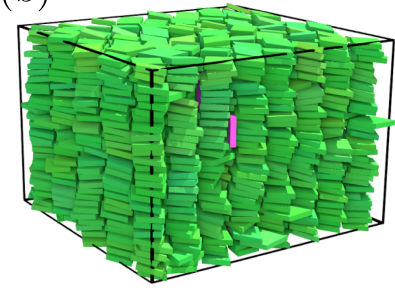

(c)

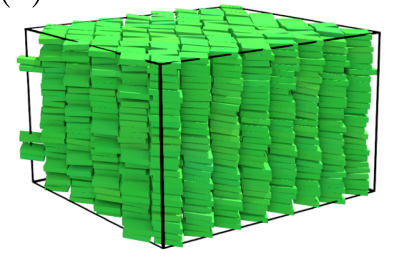

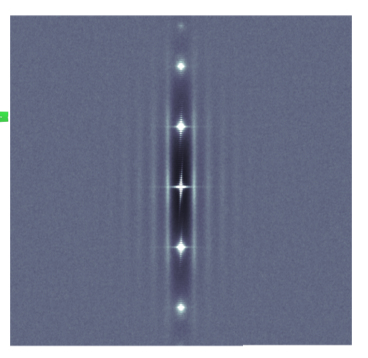
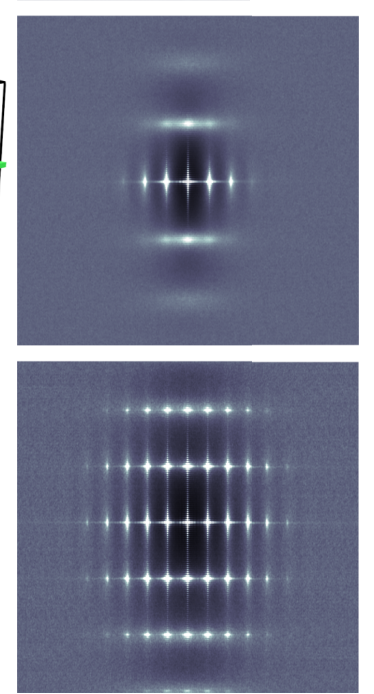

FIG. 4. Left: Representative configurations of hard rhombic platelets with reduced width $W / L=2 / 3$ and thickness $T / L=1 / 9$ for the smectic (a), columnar (b), and rhombic crystal (c) phases. The particles are colored according to the absolute value of the direction of their short axis. Right: Diffraction patterns corresponding to the phases to the left, with the vertical axis being along the $T$-nematic director and the horizontal axis perpendicular to it.

upon increasing the packing fraction, a (weakly) first-order phase transition from the isotropic to the nematic phase, followed by a (weakly) first-order phase transition to the smectic

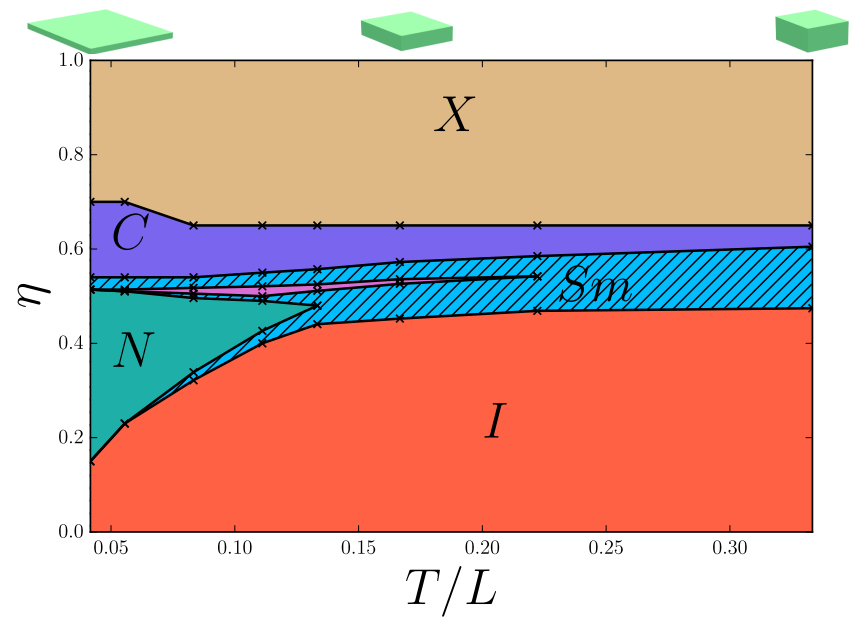

FIG. 5. Phase diagram, of hard rhombic platelets with width-to-length ratio $W / L=2 / 3$, in the packing fraction, $\eta$-reduced thickness, $T / L$ representation, determined from $N P T$ expansion runs. At the top of the diagram, and from left to right, the rhombic platelet is shown for increasing reduced thicknesses corresponding to the $x$-axis. The volume of the depicted platelets is fixed. The stable phases are coloured in the phase diagram as follows: orange: isotropic phase $(I)$, green: nematic phase $(N)$, pink: smectic phase $(S m)$, purple: columnar phase $(C)$, brown: crystal phase $(X)$, and blue: two-phase coexistence regions. phase, and another one to the columnar phase, and a continuous transition to the crystal phase. For thickness $T / L<0.083$, the smectic phase vanishes completely. It is also expected that the isotropic phase vanishes as $T / L \rightarrow 0$.

In addition, we investigated the possibility of the smectic phase being metastable. To this end, we carefully checked if the smectic phase spontaneously forms upon expanding the space-filling rhombic crystal phase, as well as upon compressing the isotropic fluid phase using MC simulations in the $N P T$ ensemble for $N \approx 2000$ rhombic platelets, with $T / L=1 / 9$. Upon compressing the isotropic fluid phase, we indeed find that the smectic phase forms spontaneously, thereby lending strong support that the smectic phase is stable. Upon compression, long-lived columnar clusters are also formed. However, we were not able to recover a bulk columnar phase upon compressing the smectic phase further, at least within the simulation time that we considered.

Furthermore, we performed event-driven molecular dynamics (EDMD) simulations of rhombic particles with $T / L$ $=1 / 9$, in the $N V T$ ensemble. For $N \approx 2000$ particles, simulations close to the isotropic-nematic and nematic-smectic coexistence showed strong spinodal-like fluctuations in the local nematic and smectic order parameters, respectively. This accompanied by the small "jumps" in the equations of state leads us to the conclusion that these transitions are weakly first order. We also find that the columnar phase melts into a smectic phase at a pressure $\beta P V_{p} \approx 7.3$, which is close to the coexistence pressure found from the MC expansion, but we find significant finite size effects for this transition, even for a columnar phase consisting of $20 \times 52$ columns, i.e., $N$ $=52000$ particles.

Although the hard rhombic platelets have a fundamentally different shape from, e.g., oblate hard spherocylinders (OHSCs) of Ref. 10, preliminary simulations show striking agreement, at least quantitatively, in the phase behavior of the two-particle shapes. Unlike the OHSC, though, we also find a smectic phase for reduced thickness $0.083<T / L<0.17$ and for packing fractions $\eta \in(0.5,0.52)$. We speculate that the smectic phase is entropically stabilized by the non-circular shape of the rhombic platelets as well as the large flat facets.

\section{SUMMARY AND OUTLOOK}

Using Monte Carlo simulations, we determined the phase diagram of hard rhombic platelets with width-to-length ratio, $W / L=2 / 3$, as a function of the plate thickness, $T / L$. We measured the equations of state, diffraction patterns, as well as the nematic and smectic order parameters, and determined the phase boundaries from NPT expansion runs. We verified the type of the various phases by visual inspection of the particle configurations. In addition, we investigated the presence of metastability by performing compression runs, upon which a bulk smectic phase is formed, and EDMD simulations in the $N V T$ ensemble. Our MC simulations as well as EDMD simulations, provide strong support for a stable columnar and smectic phase in a system of hard-rhombic platelets, but it is hard to pinpoint exactly the phase transition due to severe finite-size effects and slow equilibration rates, as the free energies of the two phases are likely very close. This will also hamper 
the determination of the phase boundaries using free-energy calculations.

The phase behavior of rhombic platelets, presented here, is compatible with that of other platelet-like particles found in the literature, such as cylinders, ${ }^{6,57}$ cut spheres, ${ }^{9,11}$ and oblate hard spherocylinders. ${ }^{10}$ Unlike the aforementioned particle shapes, the non-circular symmetry and flat facets of rhombic platelets lead to the presence of a stable smectic phase. The smectic phase is stable in a narrow density regime but could possibly be stabilized in a wider regime for different width-to-length ratios of $W / L$.

Generally, a biaxial phase is expected for anisotropic particles when the quantity $v=T / W-W / L \approx 0,{ }^{58}$ where $T<W<L$ are the sizes of the three main particle axes. For the rhombic platelets presented here, we find that a biaxial nematic phase is present for extremely thin platelets, $T / L<0.05$, corresponding to $v \approx-0.6$. Our preliminary results, as well as those found, e.g., in Ref. 59 on brick-like particles, indicate that the stability of a biaxial phase also depends on an additional criterion for $T / L$, i.e., the platelets should be sufficiently thin. In future work, we intend to study the phase behavior of rhombic particles as a function of all parameters $T, W$, and $L$, focusing on the biaxial phase.

Rhombic platelets of different aspect ratios can be synthesized and used in self-assembly experiments. In this work, we have investigated what the effect of a non-circularly symmetric particle shape is on the phase behavior of plate-like particles, and how the flat facets stabilize a layered structure such as the smectic phase.

\section{ACKNOWLEDGMENTS}

N.T. and M.D. acknowledge financial support from an NWO-ECHO grant.

${ }^{1}$ L. Onsager, Ann. N. Y. Acad. Sci. 51, 627 (1949).

${ }^{2}$ J. Vieillard-Baron, J. Chem. Phys. 56, 4729 (1972).

${ }^{3}$ D. Frenkel and B. Mulder, Mol. Phys. 55, 1171 (1985).

${ }^{4}$ P. Bolhuis and D. Frenkel, J. Chem. Phys. 106, 666 (1997).

${ }^{5}$ D. Frenkel and R. Eppenga, Phys. Rev. Lett. 49, 1089 (1982).

${ }^{6}$ R. Eppenga and D. Frenkel, Mol. Phyxs. 52, 1303 (1984).

${ }^{7}$ J. A. C. Veerman and D. Frenkel, Phys. Rev. A 45, 5632 (1992).

${ }^{8}$ M. A. Bates and D. Frenkel, Phys. Rev. E 57, 4824 (1998).

${ }^{9}$ P. D. Duncan, M. Dennison, A. J. Masters, and M. R. Wilson, Phys. Rev. E 79, 031702 (2009).

${ }^{10}$ M. Marechal, A. Cuetos, B. Martinez-Haya, and M. Dijkstra, J. Chem. Phys. 134, 094501 (2011).

${ }^{11}$ M. Marechal, A. Patti, M. Dennison, and M. Dijkstra, Phys. Rev. Lett. 108, 206101 (2012)

${ }^{12}$ P. F. Damasceno, M. Engel, and S. C. Glotzer, Science 337, 453 (2012).

${ }^{13}$ J. Henzie, M. Grünwald, A. Widmer-Cooper, P. L. Geissler, and P. Yang, Nat. Mater. 11, 131 (2012).

${ }^{14}$ A. P. Gantapara, J. de Graaf, R. van Roij, and M. Dijkstra, Phys. Rev. Lett. 111, 015501 (2013).

${ }^{15}$ A. P. Gantapara, J. de Graaf, R. van Roij, and M. Dijkstra, J. Chem. Phys. 142, 054904 (2015).

${ }^{16}$ J. de Graaf, R. van Roij, and M. Dijkstra, Phys. Rev. Lett. 107, 155501 (2011).

${ }^{17}$ J. de Graaf, L. Filion, M. Marechal, R. van Roij, and M. Dijkstra, J. Chem. Phys. 137, 214101 (2012).

${ }^{18}$ S. J. Singer and R. Mumaugh, J. Chem. Phys. 93, 1278 (1990).
${ }^{19}$ C. Vega and P. A. Monson, J. Chem. Phys. 107, 2696 (1997).

${ }^{20}$ H. Graf and H. Löwen, J. Phys.: Condens. Matter 11, 1435 (1999).

${ }^{21}$ A. Haji-Akbari, M. Engel, A. S. Keys, X. Zheng, R. G. Petschek, P. PalffyMuhoray, and S. C. Glotzer, Nature 462, 773 (2009).

${ }^{22}$ P. I. C. Teixeira, A. Masters, and B. Mulder, Mol. Cryst. Liq. Cryst. Sci. Technol., Sect. A 323, 167 (1998).

${ }^{23}$ Y. Martinez-Raton, S. Varga, and E. Velasco, Phys. Chem. Chem. Phys. 13, 13247 (2011).

${ }^{24}$ A. B. L. op Reinink, S. Belli, R. van Roij, M. Dijkstra, A. V. Petukhov, and G. J. Vroege, Soft Matter 10, 446 (2014).

${ }^{25}$ S. D. Peroukidis and A. G. Vanakaras, Soft Matter 9, 7419 (2013).

${ }^{26}$ S. Dussi and M. Dijkstra, Nat. Commun. 7, 11175 (2016).

${ }^{27}$ M. Dijkstra, Adv. Chem. Phys. 156, 35 (2014).

${ }^{28}$ G. Kolbe, "Das Komplexchemische Verhalten der Kieselsaure," Ph.D. Dissertation, Friedrich-Schiller-Universität Jena, 1956.

${ }^{29}$ W. Stöber, A. Fink, and E. Bohn, J. Colloid Interface Sci. 26, 62 (1968).

${ }^{30}$ J. Zhang, Z. Luo, B. Martens, Z. Quan, A. Kumbhar, N. Porter, Y. Wang, D.-M. Smilgies, and J. Fang, J. Am. Chem. Soc. 134, 14043 (2012).

${ }^{31}$ J. J. Choi, K. Bian, W. J. Baumgardner, D.-M. Smilgies, and T. Hanrath, Nano Lett. 12, 4791 (2012).

${ }^{32}$ Z. Quan, W. S. Loc, C. Lin, Z. Luo, K. Yang, Y. Wang, H. Wang, Z. Wang, and J. Fang, Nano Lett. 12, 4409 (2012).

${ }^{33}$ M. Eguchi, D. Mitsui, H.-L. Wu, R. Sato, and T. Teranishi, Langmuir 28, 9021 (2012)

${ }^{34}$ X. Xia and Y. Xia, Nano Lett. 12, 6038 (2012).

${ }^{35}$ L. Rossi, S. Sacanna, W. T. M. Irvine, P. M. Chaikin, D. J. Pine, and A. P. Philipse, Soft Matter 7, 4139 (2011).

${ }^{36}$ H.-L. Wu, C.-H. Kuo, and M. H. Huang, Langmuir 26, 12307 (2010).

${ }^{37}$ X. Zhang, C. Dong, J. A. Zapien, S. Ismathullakhan, Z. Kang, J. Jie, X. Zhang, J. C. Chang, C.-S. Lee, and S.-T. Lee, Angew. Chem., Int. Ed. 48, 9121 (2009).

${ }^{38}$ A. R. Tao, D. P. Ceperley, P. Sinsermsuksakul, A. R. Neureuther, and P. Yang, Nano Lett. 8, 4033 (2008).

${ }^{39}$ S. C. Glotzer and M. J. Solomon, Nat. Mater. 6, 557 (2007).

${ }^{40}$ A. R. Tao, P. Sinsermsuksakul, and P. Yang, Angew. Chem., Int. Ed. 45, 4597 (2006).

${ }^{41}$ A. S. Barnard, X. M. Lin, and L. A. Curtiss, J. Phys. Chem. B 109, 24465 (2005).

${ }^{42}$ B. Wiley, T. Herricks, Y. Sun, and Y. Xia, Nano Lett. 4, 1733 (2004).

${ }^{43}$ Y. Sun and Y. Xia, Science 298, 2176 (2002).

${ }^{44}$ E. Matijevic, Acc. Chem. Res. 14, 22 (1981).

${ }^{45}$ T. Paik, D.-K. Ko, T. R. Gordon, V. Doan-Nguyen, and C. B. Murray, ACS Nano 5, 8322 (2011).

${ }^{46}$ T. Paik, T. R. Gordon, A. M. Prantner, H. Yun, and C. B. Murray, ACS Nano 7, 2850 (2013).

${ }^{47}$ T. Paik, B. T. Diroll, C. R. Kagan, and C. B. Murray, J. Am. Chem. Soc. 137, 6662 (2015).

${ }^{48}$ X. Ye, J. Chen, M. Engel, J. A. Millan, W. Li, L. Qi, G. Xing, J. E. Collins, C. R. Kagan, J. Li, S. C. Glotzer, and C. B. Murray, Nat. Chem. 5, 466 (2013).

${ }^{49}$ J. H. Conway, H. Burgiel, and C. Goodman-Strauss, "Naming archimedean and catalan polyhedra and tilings," in The Symmetries of Things (CRC Press, 2016), Chap. 21.

${ }^{50}$ N. G. Almarza, J. Chem. Phys. 130, 184106 (2009).

${ }^{51}$ E. G. Gilbert, D. W. Johnson, and S. S. Keerthi, IEEE J. Rob. Autom. 4, 193 (1988).

${ }^{52}$ P. de Gennes, Mol. Cryst. Liq. Cryst. 12, 193 (1971).

${ }^{53}$ P. de Gennes and J. Prost, The Physics of Liquid Crystals (Oxford University Press, 1993).

${ }^{54}$ K. Kobayashi, Phys. Lett. A 31, 125 (1970).

${ }^{55}$ W. L. McMillan, Phys. Rev. A 4, 1238 (1971).

${ }^{56}$ M. Cifelli, G. Cinacchi, and L. De Gaetani, J. Chem. Phys. 125, 164912 (2006).

${ }^{57}$ R. Blaak, D. Frenkel, and B. M. Mulder, J. Chem. Phys. 110, 11652 (1999).

${ }^{58}$ B. Mulder, Phys. Rev. A 39, 360 (1989).

${ }^{59}$ S. Dussi, "When shape is enough: From colloidal spheres to twisted polyhedra, from icosahedral to chiral order," Ph.D. thesis, University of Utrecht, 2016, http://web.science.uu.nl/scm/Theses/Dussi.pdf. 